in the cystadenomatous group compared with 20 in the group of embryonal carcinoma or malignant teratoma, 17 innocent dermoid cysts, 15 dysgerminomata, and 8 granulosa-cell tumours. Abdominal pain was the commonest symptom and also the commonest first symptom in Lindfors's patients. Three-quarters of the children had a palpable abdominal swelling, though the mass was not always recognized when the patient was first seen-an indication of the more difficult diagnostic problems in this age group. Indeed, in the group as a whole there was an average delay of three to four months from the time of first examination to the time of diagnosis. When no tumour is palpable Lindfors stresses the value of a plain abdominal $x$-ray examination, which may show teratomatous calcification and may rule out other disorders. Nevertheless, a preoperative diagnosis was not always possible and operation was performed in $21 \%$ of cases on a provisional diagnosis of appendicitis.

A surgeon may find it difficult to decide how extensive his operative treatment should be, especially when he comes on the ovarian tumour unexpectedly. The treatment in 27 cases of innocent neoplasm in Lindfors's series was salpingooophorectomy in 17 and cystectomy in only 10. A simple cystic ovarian tumour is probably (though not certainly) innocent, while dermoid cysts are readily recognizable if shelled out from the ovary and inoised, when their typical contents are seen. Conservation of ovarian tissue is most important in children, and initially cystectomy is generally preferable to either oophorectomy or salpingo-oophorectomy for probably innocent lesions. But lesions that are possibly malignant call for removal of all the affected ovary. When this seems completely to have removed the macroscopic tumour no more need be done, apart from careful follow-up. A more extensive malignant lesion, with spread to the opposite ovary, the uterus, or elsewhere, can seldom be dealt with satisfactorily by other than total hysterectomy and bilateral salpingo-oophorectomy, and not always then. In Lindfors's series $52 \%$ of the children with malignant lesions survived alive and well for two years. After that there were no fatalities in $74 \%$ of the survivors who were followed up for five years, and none in $30 \%$ who were followed up for 10 years.

Awareness that little girls may develop ovarian neoplasms together with greater insistence on careful abdominal and rectal examination in any child with abdominal pain will lead to earlier and more reliable diagnoses, which are so important to ultimate survival.

\footnotetext{
1 Forshall, I., Archives of Diseases in Childhood, 1960, 35, 17.

2 Thatcher, D. S., Surgery, Gynecology and Obstetrics, 1963, 117, 477.

${ }^{3}$ Lindfors, O., Annales Chirurgiae et Gynaecologiae Fenniae, 1971, 60, Supplement 177.
}

\section{Screening for Mental Disorder}

Psychiatric screening was being tried fifty years ago. "Every effort should be made at the time of enlistment to ascertain the nervous and mental conditions of candidates both from their previous histories and from their present condition. Only fit men ... should be enlisted into the regular Army." In the second world war, too, efforts were made to exclude unsuitable people from war service and from air crews ${ }^{2}$ as well as to choose people for training as officers.

This type of screening is designed, as is quarantine, to protect society from the individual. In a newer sense screening means the discovery of presymptomatic disease or of disease in its early stages, and it is employed primarily for the benefit of the individual. But initial enthusiasm, largely generated by the success of mass miniature radiography in detecting early cases of pulmonary tuberculosis, has waned. It has been succeeded by a more cautious phase marked by the publication in 1968 of two authoritative reports. ${ }^{34}$ The first enunciated ten principles as guides to planning casefinding. The second subjected ten widely used screening procedures to rigorous analysis and found that all of them could be criticized on the grounds that too little is known of the natural history of the diseases concerned, or that the best available methods of diagnosis and treatment are unsatisfactory, or that the poor haul hardly justifies the cost of trawling.

Since then controversy has erupted over, for example, screening for phenylketonuria, in which for ethical reasons a controlled trial of dietary treatment has not been carried out. Ironically, too, the arrangements for random detection of tuberculosis by mass radiography are being phased out. ${ }^{5}$ Nevertheless programmes of screening for a single disease or a multiplicity of them go ahead, particularly in the United States and in a few places in Britain. Screening for hypertension, at least in middle-aged men, has also staged a comeback. ${ }^{6}$

Any proposal to introduce an entirely new screening programme is likely to be resisted and before being accepted will have to be judged by the ten principles of J. M. G. Wilson and G. Jungner ${ }^{3}$ (or something like them). According to these the disorder to be screened should be important and it should have a recognizable latent or presymptomatic stage; the disease should be treatable and facilities for diagnosis and treatment should be available; tests for the disorder should be reliable and acceptable; the natural history of the condition should be understood; there should be a cut-off point between those found to need and those found not to need treatment; and the cost-effectiveness of screening should compare with that of other medical services.

Recently M. R. Eastwood ${ }^{7}$ assessed psychiatric disorder as a candidate for screening. He found it at present unsuitable in almost every respect and concluded: "The problems of definition of diagnostic categories, knowledge of the natural history of neurosis, and effectiveness of treatment require solving." In addition, there are disagreements about diagnosis-notably between British and American psychiatrists - of the psychoses, 89 admittedly only a small proportion of the whole. The disparities exist too between textbook descriptions of neurosis, which are apt to give the hospital view, and the facts of minor nervous illness as general practitioners see them. Who can say when exactly the worrier stumbles over the line into an anxiety state or when moodiness deserves the name depression? Exactly where is the presymptomatic stage in these disorders? General practitioners already provide diagnostic facilities and treatment for $90 \%$ of patients presenting with psychiatric symptoms. ${ }^{10}$ If screening were to provide more patients, could they be treated?

Eastwood reviews the known facts about the distribution of psychiatric disorder and shows how with community surveys and general practice surveys we have come some way towards establishing prevalence rates in the population at large. These rates may be similar in places as far apart 
as Baltimore and London. He also describes the instruments suitable for use in psychiatric screening-questionnaires long and short and interviews standardized or not-and stresses the need for such instruments to be valid, reliable, and stable. In an important section of his paper the author describes a psychiatric screening survey which formed part of a multiple screening programme carried out in one London borough. He achieved only a $70 \%$ acceptance rate, which must limit any conclusions that can be drawn. The screening test used was a selection of 20 items from the Cornell Medical Index. People scoring more than 5 were given a standardized clinical interview. There were $19 \%$ false negatives and $6 \%$ false positives. Moreover, interesting differences appeared between the assessments of the research psychiatrist and of five general practitioners taking part in the project.

In psychiatry the major research effort so far has been directed to psychoses, the severe neuroses, and alcoholism. Eastwood has made a case for devoting more attention to the minor psychiatric disorders and offers some suggestions on how that might be done. Even so, there is no dissenting from his conclusion that "for the present, as with some other forms of morbidity, screening for psychiatric disorder must remain at the experimental phase and is not ready for inclusion in the medical services."

\footnotetext{
${ }^{1}$ Report of the War Office Committee of Enquiry into 'Shell Shock', Cmnd.

1734 . London, H.M.S.O., 1922 .
2 The Work of Psychologists and Psychiatrists in the Services-Report of an Expert Committee. London, H.M.S.O., 1947.

3 Wilson, J. M. G., and Jungner, G., Principles and Practice of Screening for Disease. Geneva, W.H.O. (Public Health Papers No. 34), 1968.

4 McKeown, T., et al., Screening in Medical Care. London, Oxford University Press, for the Nuffield Provincial Hospitals Trust, 1968.

5 British Medical fournal, 1970, 1, 57.

- Rose, G. A., Health Trends, 1971, 3, 2.

7 Eastwood, M. R., Psychological Medicine, 1971, 1, 197.

8 Kramer, M., in Proceedings of the Third World Congress of Psychiatry. Montreal, McGill University Press, 1961.

- Kendell, R. E., British Fournal of Hospital Medicine, 1971, 6, 147.

10 Shepherd, M., Cooper, B., Brown, A. C., and Kalton, G. W., Psychiatric Illness in General Practice. London, Oxford University Press, 1966.
}

\section{Orbital Varices}

Congenital venous malformations of the orbit are not uncommon. They' nearly always present in childhood or in youth with a proptosis which is aggravated by pressure on the head or neck, and they may be associated with venous dilatations in the neighbouring skin and conjunctiva. Pulsation in such cases normally signifies an arteriovenous shunt, but it may occur in the absence of arteriovenous communication and be attributable to the transmission of cerebral pulsation through an enlarged sphenoidal fissure. Straight $x$-ray examination may provide the diagnosis when the affected orbit is shown to be enlarged, and particularly if there are any concentric rings of calcification from a phlebolith. But the particular value of orbital phlebography in delineating venous malformations within the orbit has been shown in 12 cases of orbital varices described by G. A. S. Lloyd and his colleagues. ${ }^{1}$

Orbital phlebography has been increasingly practised in recent years. It is now usually performed through the frontal rather than the angular vein and after compression over the brow and jugular veins. Compared with carotoid angiography the procedure is relatively simple. It is often done under local anaesthesia and the mortality is nil. As a guide to diagnosis it is valuable not only in frank venous mal- formations but also in many of the other ambiguous disorders presenting merely as symptom-free unilateral exophthalmos.

1 Lloyd, G. A. S., Wright, J. E., and Morgan, G., British Fournal of Ophthalmology, 1971, 55, 505.

\section{Continuous Fetal Heart Rate}

Until recently, because of instrumentation problems, continuous recording of the fetal heart rate was more suited to research than clinical practice. Now with technical improvements the equipment is available for use in the labour ward. The potential advantages of the continuous record to the clinician in terms of the reliable and early detection of fetal asphyxia in labour are considerable. But the record needs to be interpreted differently in certain respects from that of the averaged rate recorded by the midwife when she listens between contractions. The continuous record provides information about the fetal heart rate during uterine contractions, when slowing is common and does not necessarily signify fetal asphyxia.

In $1963 \mathrm{R}$. Caldeyro-Barcia and co-workers ${ }^{1}$ studied in considerable detail the changes in fetal heart rate of an anencephalic fetus in response to uterine contractions of variable frequency and intensity. They showed that there was a direct relationship between the intensity of the contractions and the amplitude, duration, and timing of the transient fetal bradycardia (dips or decelerations) caused by the contractions. The more intense and frequent the contractions became, the later was the onset of the bradycardia after each contraction, until eventually, when the interval between the point of maximal uterine pressure and of the slowest heart rate exceeded 20 seconds (type II dip in heart rate), the fetus died. This work has frequently been confirmed and forms the basis of all subsequent studies on the evaluation of the continuous fetal heart rate.

Interpretation of changes in the continuous fetal heart rate would be simple if the diagnosis of fetal asphyxia depended on whether type II dips were present or not. E. H. Hon $^{2}$ has shown that asphyxia due to compression of the umbilical cord causes bradycardia early in the contraction phase-a type I dip-and cases have been described in which only type I dips were recorded until shortly before fetal death. ${ }^{3} 4$ Moreover the issue is complicated by the fact that it is uncommon for any two consecutive uterine contractions to produce the same change in heart rate. The amplitude of dips is not necessarily a reflection of the severity of fetal asphyxia, and it is now recognized that shallow type II dips with a slow recovery (late deceleration of $\mathrm{Hon}^{5}$ ) are commonly accompanied by fetal acidosis and a baby born in poor condition. Loss of the normal fluctuations in fetal heart rate, often referred to as "smoothing,"5 or "loss of beat-to-beat variation"6 and tachycardia between contractions are frequent accompaniments of fetal asphyxia.

Attempts have been made to quantitate several features of the continuous fetal heart rate known to be associated with fetal asphyxia into a single expression and relate it to a period of time. ${ }^{78}$ Recently T. Shelley and R. H. Tipton ${ }^{9}$ have combined the independent measurements of frequency, duration, and amplitude of fetal bradycardia by measuring the sum of the products of fall in heart rate over a one-hour period. The figure arrived at is termed the "dip area" and 